\section{Proto-Language as a Structurer and Enhancer of Perception}

\author{
James Scott ${ }^{1}$, Robert Foley ${ }^{2}$, Mirjana Bozic ${ }^{1}$
}

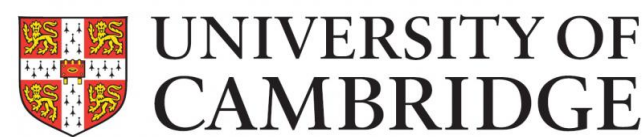

1 Department of Psychology

2 Leverhulme Centre for Human Evolutionary Studies University of Cambridge, Cambridge, UK

Contact: jhs74@cam.ac.uk

\section{Background}

Language evolution is hotly debated, though most likely a protracted and mosaic process ${ }^{1}$. The present study investigated factors which might have contributed to the evolution of semantics and formation of concepts. We hypothesised that noncommunicative functions of language (NCF), which refer to the ability of language to alter and enhance cognition ${ }^{2}$, might have provided one important selective advantage. In addition, iconicity or the non-arbitrariness of some form-meaning mappings ${ }^{3}$, may have provided a foothold for the development of semantics by providing links between sound and meaning. To investigate NCF and iconicity as possible adaptive advantages and ontogenetic facilitators in the evolution of semantics, we trained participants on novel semantic categories that were paired with either iconic labels, non-iconic labels, or no labels. Match to Sample (MTS) task was then used to assess whether labelling and iconicity manipulations affected visual recognition of category members.

\section{Conditions}

This was a between-subjects design, with each participant exposed to only one condition. The purpose of Training was for participants to learn the novel semantic categories of aliens, and their associated labels. The purpose of MTS was to assess rapid visual recognition of category members, and how this was facilitated by labels. For MTS, the online/offline subconditions

\begin{tabular}{|c|c|c|}
\hline \multirow{3}{*}{1 Iraining Condition } & Match to Sample Condition & meant th \\
\hline & 1a Iconic online & \\
\hline & 1b Iconic offline & either present \\
\hline \multirow[t]{2}{*}{2 Non-iconic label } & 2a Non-iconic online & $(1 a \& 2 a)$ or $r$ \\
\hline & 2b Non-iconic offline & $(1 b \& 2 b)$ at \\
\hline 3 No-label & 3 No-label & $\begin{array}{l}\text { Deginning } \\
\text { each trial. }\end{array}$ \\
\hline
\end{tabular}

\section{Methods}

Stimuli: Visual stimuli were 625 unique 'aliens' which varied along 4 dimensions (spoke number, spikiness, body size, and colour). Aliens were generated using a tensor, and segregated into 2 categories; see right for 2D example. Auditory stimuli consisted of 2 iconic and 2 non-iconic pseudoword labels.

Participants: 141 neurotypical adults with good command of English, recruited online.

Procedure: Online game, with 2 blocks: Training and Match to Sample (MTS). Data: RTs and accuracy. In Training (180 trials), adapted from ${ }^{4}$, participants learnt alien categories by interacting with them and receiving accuracy feedback (with auditory label presentation in the label conditions). In MTS (200 trials), participants were presented with a novel target and 2 distractor aliens (1 from each category); and asked to match categorically. In online conditions participants heard the relevant label before stimuli presentation, to distinguish learning from online effects.

Data Collection and Analysis: Participants were recruited through Prolific ${ }^{5}$ and Sona ${ }^{6}$; tested on PsyToolkit ${ }^{7,8}$. N=146, 13 excluded. RTs were analysed via independent threeway mixed ANCOVAs, with age as a covariate. Accuracy was coded binarily, and analysed via logistic regression with age as a covariate. Data were analysed in $\mathrm{JASP}^{9}$.

\begin{tabular}{|l|l|l|}
\hline \multicolumn{1}{|c|}{ Condition } & \multicolumn{1}{c|}{ Category 1 } & \multicolumn{1}{c|}{ Category 2 } \\
\hline Iconic & Glulge (/glıld3/ & Skysk (/ski:sk/) \\
\hline Non-Iconic & Stoise (/storz/) & Phrav (/fræv/) \\
\hline No Label & - & - \\
\hline
\end{tabular}

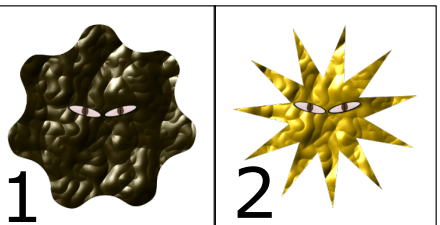

4. Results
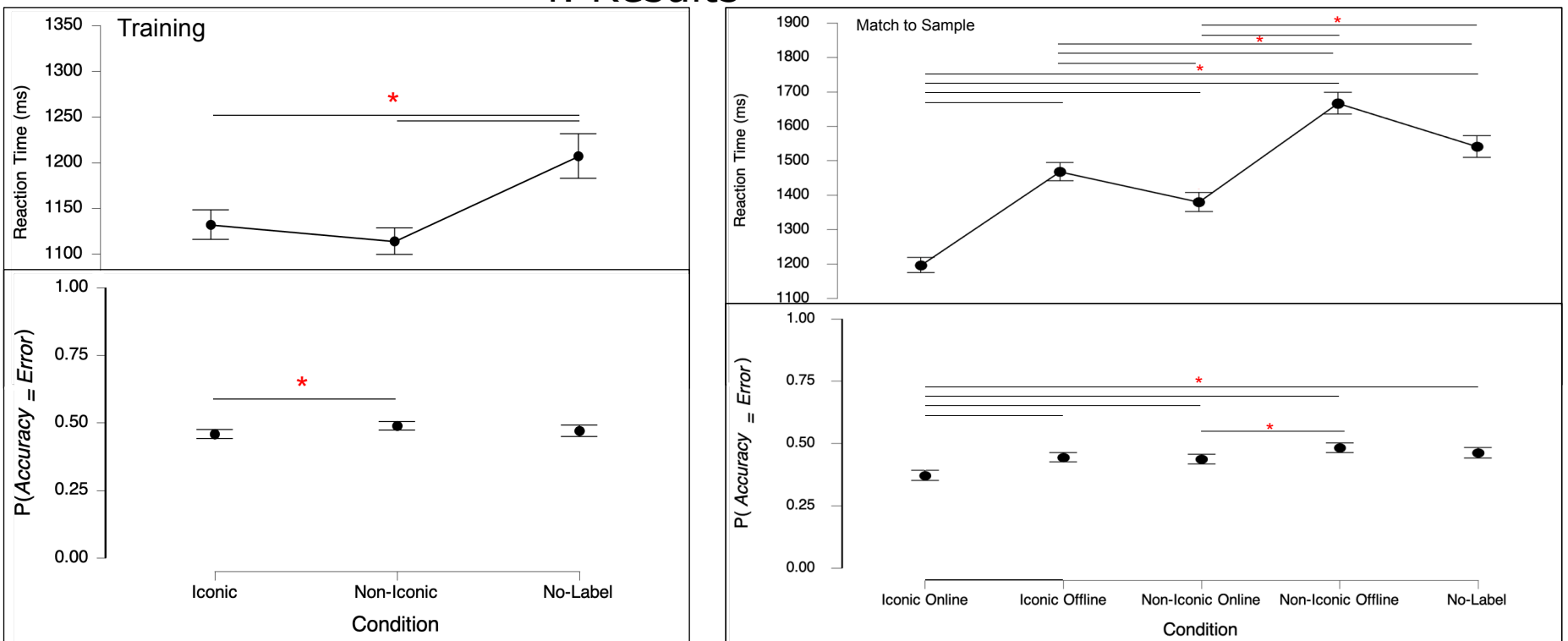

Training: Participants in the no-label Match to Sample: Participants in online conditions were significantly faster than those in offline condition were significantly slower than those conditions. Participants in iconic conditions were significantly faster than those in all other in label conditions. Participants in the iconic conditions. Participants in the iconic online condition were thus significantly faster than those in label condition were significantly more any other condition. Participants in the iconic online condition were also significantly more accurate than those in the non-iconic label accurate than those in any other condition. Participants in the non-iconic online condition were condition.

\section{Conclusions}

- Labels facilitate the learning of nove semantic categories

- This is enhanced by iconicity

- These learning advantages facilitate rapid visual recognition in MTS

- These effects are further exaggerated by online presentation of labels

- These effects were observed with very little training also significantly more accurate than those in the non-iconic offline condition 